\section{An evaluation of public recognition and attitude towards depression}

\section{To the Editor}

I have read with interest the article by AL Jadaani et $\mathrm{al},{ }^{1}$ they carried out an interesting survey-based study. This work aims to measure the degree of public awareness and attitude towards depression. Lately, mental health has attracted great interest, particularly during the recent COVID-19 pandemic given emerging reports suggestive of an increased risk of mental illnesses during the pandemic.

Several studies have linked psychological distress to obesity, alcohol and substance abuse, poor compliance to medications, an increased risk of stroke and coronary artery disease, and poor cancer prognosis. It has also been established that enhanced mental health strengthens overall well-being and improves intrinsic motivation, productivity, and creativeness. It is an integral element in social cohesion, stability, empowerment of the workforce and economic strength of the communities.

Depression is one of the most prevalent mental illnesses and it has been associated with lack of interest, restricted activity and missing working days, with an inherent social stigma as suggested by the authors, based on significant rate of survey responses that is attributing depression to weakness in faith, defaults in personality and evil eye.

The authors showed that majority of the public were not aware of approved therapeutic approaches for resistant depression, but they did not object on forming friendships and establishing work relationships with people suffering from depression which indicates interest and tendency of the community to provide social support to patients with mental illnesses.

However, the authors' survey results cannot lead to an accurate conclusion since the recruitment strategy is likely to be associated with sampling bias. I agree with the authors that the survey covers majority of younger population on higher educational levels and possibly greater interest in mental health disorders compared to general population. The survey is also restricted to one region limiting the generalizability and validity of the results.
In this context, robust surveys are needed to collect data and design public awareness campaigns and educational interventions. Further analysis is also required down the road to evaluate and improve the strategies implemented.

\section{Muneera AL Hussaini Department of Internal Medicine Prince Sultan Military Medical City Riyadh, Kingdom of Saudi Arabia}

Saudi Med J 2022; Vol. 43 (2): 218 doi: 10.15537/smj.2022.43.2.20210928

\section{Reply from the Author}

First of all, the authors would like to thank and show appreciation to Dr. Muneera AL Hussaini upon the commentary (An evaluation of public recognition and attitude towards depression) regarding our article.

Depression is one of the most common mental illnesses around the globe, our study aimed to investigate the degree of public awareness, beliefs, and attitudes regarding major depression and available treatment options in Hail region, Saudi Arabia.

The authors agree on that the study recruitment method can be designed in a better way, we also agree that further studies with larger sample sizes, more variables, and coverage of all regions in Saudi Arabia are warranted to address these limitations.

The authors stated in the article that mental health promotion and educational efforts are necessary to improve mental health literacy and clarify misconceptions regarding depression in the general public.

\section{Ahmed H. ALJadani Internal Medicine Department College of Medicine, University of Hail Hail, Kingdom of Saudi Arabia}

\section{References}

1. ALJadani AH, Alshammari SN, Alshammari KA, Althagafi AA, AlHarbi MM. Public awareness, beliefs and attitude towards depressive disorders in Saudi Arabia. Saudi Med J 2021; 42: 1117-1124. 